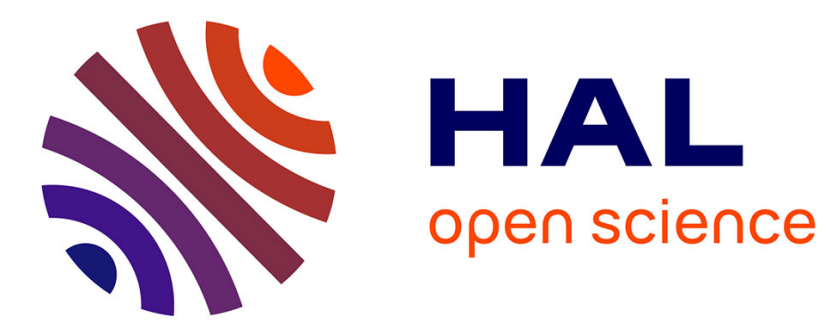

\title{
THEORIES VERSUS EXPERIMENTS IN THE SPIN GLASS SYSTEMS
}

\author{
André Blandin
}

\section{To cite this version:}

André Blandin. THEORIES VERSUS EXPERIMENTS IN THE SPIN GLASS SYSTEMS. Journal de Physique Colloques, 1978, 39 (C6), pp.C6-1499-C6-1516. 10.1051/jphyscol:19786593 . jpa-00218086

\section{HAL Id: jpa-00218086 https://hal.science/jpa-00218086}

Submitted on 1 Jan 1978

HAL is a multi-disciplinary open access archive for the deposit and dissemination of scientific research documents, whether they are published or not. The documents may come from teaching and research institutions in France or abroad, or from public or private research centers.
L'archive ouverte pluridisciplinaire HAL, est destinée au dépôt et à la diffusion de documents scientifiques de niveau recherche, publiés ou non, émanant des établissements d'enseignement et de recherche français ou étrangers, des laboratoires publics ou privés. 


\section{THEORIES VERSUS EXPERIMENTS IN THE SPIN GLASS SYSTEMS}

\section{A. Blandin}

Laboratoire de Physique des Solides, Bâtiment 510, Université Paris-Sud, 91405 Orsay, France

Résumé.- Après avoir résumé les principales propriêtés expérimentales des verres de spin (chaleur spécifique, température critique, phénomènes d'hystérêsis et de métastabilité), nous montrons comment les théories existantes phénoménologiques et de champ moyen se sont développées. Le concept de frustration, associê à une thêorie de gauge s'avère prometteur, mais il reste beaucoup à faire pour comprendre cette nouvelle transition de phase.

Abstract.- After a rapid description of the main experimental properties of spin glasses (specific heat, critical temperature, hysterisis and metastability), we show how the actual theories (phenomenology, mean field theory) are being developed. The concept of frustration, and its link to gauge theories seems promising, but a lot of work has to be done in order to understand this new phase transition.

1. INTRODUCTION.- The expression "spin glass" appeared about ten years ago, in order to describe the properties of dilute magnetic impurities in normal metals, canonical examples being CuMn or AuMn. In fact, it was an old problem : the first experiments began long time ago; the recognition of a new kind of magnetism, about twenty years ago, led Friede1 to think of a "freezing" of the magnetic disorder, linked to the oscillatory exchange interactions and giving rise to a continuous distribution of static molecular fields. We wrote : "the spin disorder is "frozen" at low temperatures" /1/. In spin glasses there is no spatial long range order and the questions arises : in those conditions is there an "order parameter", is there a phase transition and what are their characteristic behaviours ? I sha11 come back to these questions several times in this review paper.

In the same alloys, various physicists (de Nobe1, Van den Berg ...) observed a minimum in the resistivity $\rho(T)$. It appeared later that this was a "one impurity" effect, the Kondo effect, which is characterized by a temperature $T_{K}$. In order to study the spin-glass behaviour, without mixing it with the Kondo problem, we need temperatures much larger than $\mathrm{T}_{\mathrm{K}}$. Happily, $\mathrm{T}_{\mathrm{K}}$ is very small in CuMn or AuMn and the lower limitation for the concentration is not drastic.

The concentration of impurities should not be too large. In that case near neighbour interactions are dominant and give rise to an ordinary magnetic phase : an example is $\mathrm{Au}_{4} \mathrm{Mn}$, which is spatially ordered and ferromagnetic. The two limitations are not severe and experiments can be done over several decades of concentrations.

What are the main properties of spin glasses? The first experiments were done at high temperatures by Nêel and Weil giving a Curie paramagnetic behaviour with a large Curie temperature $T_{p}$, of the order $\mathrm{x} \times 10^{3} \mathrm{~K}, \mathrm{x}$ being the concentration of impurities. Kittel and coworkers $/ 2 /$ found the same high temperature results but observed a broad maximum of the susceptibility $X(T)$ for a temperature roughly proportional to the concentration and of the order $T_{P}$.

A striking feature of the spin glasses as measured by Zimmerman and Hoare is the behaviour of the extra specific heat due to the impurities which is a linear function of $\mathrm{T}, \mathrm{c}=\gamma \mathrm{T}, \gamma$ being independent of the concentration and much larger than the ordinary linear term of the normal metal $/ 3 /$.

At sufficiently low temperatures, there exist multiple evidences of training and hysterisis, with small or large jumps in the $\mathrm{m}(\mathrm{H})$ curves.

Though there is no experimental proof of long range order (in space) a critical temperature $\mathrm{T}_{\mathrm{SG}}$ shows up. There is a "cusp" in the susceptibility $\chi(T)$ in small fields for $\mathrm{T}={ }^{\mathrm{T}} \mathrm{SG} \cdot \mathrm{T}_{\mathrm{SG}}$ is also proportional to the concentration. This seems the experimental proof for a new low temperature phase, the spin-glass phase.

In this review paper, we shall develop these characteristic properties and discuss how theory (or theories) are able (or not able) to explain them. 
2. HAMILTONIAN OF REAL SPIN GLASSES AND SIMPLE CONSEQUENCES.- Non magnetic impurities (at distance $R$ apart) interact via the conduction electrons, giving rise to an energy of interaction which behaves at large distances as :

$E_{12} \simeq E_{0} \frac{\cos \left(2 k_{F} R+\phi_{0}\right)}{\left(k_{F} R\right)^{3}}$

These are the Friedel oscillations.

Similarly, magnetic impurities interact via the conduction electrons giving rise to Heisenberg interactions $\mathrm{H}_{12}$ which behave for large $\mathrm{R}$ as :

$\mathrm{H}_{12} \simeq \mathrm{J}_{\mathrm{o}} \frac{\cos \left(2 \mathrm{k}_{\mathrm{F}} \mathrm{R}+\phi\right)}{\left(\mathrm{k}_{\mathrm{F}} \mathrm{R}\right)^{3}} \overrightarrow{\mathrm{s}}_{1} \cdot \overrightarrow{\mathrm{s}}_{2}$

If the interaction $\mathrm{J}_{s d}$ between one magnetic impurity and the sonduction electrons is small, then $J_{0} \approx \frac{\mathrm{J}^{2} \text { sd }}{\mathrm{E}_{\mathrm{F}}}$ and $\phi=0$. This is the RKKY interaction (Ruderman-Kittel-Kasuya-Yosida), which is certainly valid for Rare-Earth impurities. For transitional impurities, the description in terms of virtual bound states (Friede1, Anderson) is better $: \phi \neq 0$ (this is not important for the following discussion) but $J_{0}$ is much larger.

The total Hamiltonian describing the system is :

$H=\sum_{(i, j)} H_{i j}$

This Hamiltonian shows that the interactions depend upon the positions of the spins and are not independent. Thus, in a pure magnetic metal (Gd for example), one must keep the true interaction with its oscillatory behaviour, which gives rise, by Fourier transform, to the Kohn anomaly.

On the contrary, in dilute alloys, one may hope that the magnetic atoms being randomly distributed in space (with perhaps a small short range spatial order), the exchange interaction (for large values of $R$ ) will be similar to random interactions :

$\pm \frac{J_{0}}{\left(k_{F}\right)^{3}}$

with signs + or - at random.

There is no theoretical proof of this fact, but experiments, as we shall see, validate this statement. A necessary condition is certainly that $\mathrm{k}_{\mathrm{F}}^{-1}$ (which is of the order of the lattice constant a) should be much smaller than the distance between spins. At short distances, equation (4) has no validity. a) Percolation and the effective interactions.- One can introduce the concept of percolation in this problem /4/: let us suppose that the interactions are zero for $R$ larger than a given value $R_{c}$. Then, there is a critical concentration $x_{c}$, such that for $\mathrm{x}<\mathrm{x}_{\mathrm{c}}$ there is no infinite cluster of interacting spins. On the contrary, for $x>x_{c}$ an infinite cluster shows up. From numerical results on various lattices and various kinds of increasing neighbouring (nearest neighbours, next nearest neighbours ...), one can define a universal number $\mathrm{n}_{0}$ which characterizes the occurence of an infinite cluster :

$\mathrm{n}_{\mathrm{o}} \simeq 2.6$. Then, we must have,

$x_{c}=\frac{3 n_{o} v_{o}}{4 \pi R_{c}^{3}}$

$v_{0}$ being the atomic volume.

Equation (5) defines a characteristic length $\dot{R}_{\text {o }}$ such that :

$x_{c}=\left(\frac{R_{o}}{R_{c}}\right)^{3}$

Reciproca11y, for a given concentration $x$, one can define a length :

$R(x)=R_{0} x^{-1 / 3}$

and divide the interactions in two parts :

$\mathrm{R}<\mathrm{R}(\mathrm{x})$ : The interactions are strong and the spins strongly correlated but these interactions are una$b$ le to produce collective phenomena; $R>R(x)$ : The interactions are weaker but they are the only ones which are able to produce collective phenomena.

These considerations give for the correlation function $|S(0) S(R)|$ at $T=0$ the qualitative behaviour of figure 1 (a). For the Ising model, Klein and Brout $/ 5 /$ have calculated a more precise curve with a tail for $R>R(x)$. (figure 1 (b)).

The conclusions are the following :

At high temperatures, all exchange interactions have importance and the nearest ones are dominant.

At low temperatures, on the contrary, single atoms, pairs, triplets ... with $R<R(x)$ behave as rigid magnetic moments. They interact through the long range part given by equation (4).

This description, which is a very crude one, shows that in order to discuss the low temperature phase, one should modify the interaction $J(R)$ into effective ("renormalized") $\Im(R)$. During this process, the strongest interactions are strongly reduced; on 
the contrary, the weak interactions remain nearly the same. A good description of the real spin glasses should give a quantitative answer to this qualitative description.

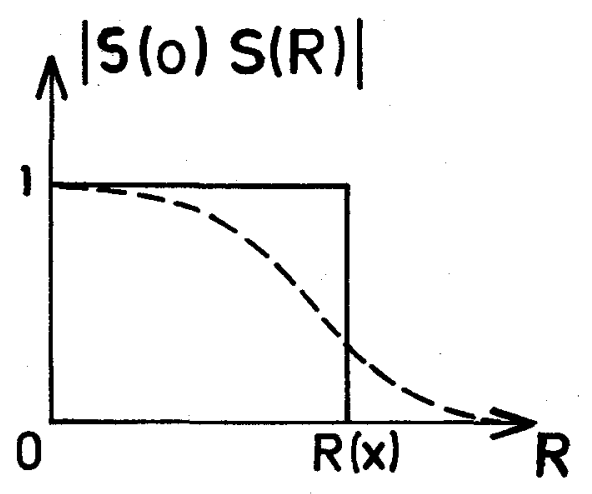

Fig. 1 : Correlation function $|S(0) S(R)|$

(a) rough estimation

(b) result of Klein and Brout /5/

A direct consequence of this discussion concerns the high temperature behaviour of the susceptibility proportional to $x$ with a paramagnetic Curie temperature $T_{p}=x \tilde{J}_{0}$ where :

$\tilde{J}_{0}=\overline{\sum_{j}\left(R_{i j}\right)}$

and the average is over the spins $i$. In (8), all interactions $R<R(x)$ and $R>R(x)$ are taken into account. This behaviour is valid when $k_{B} T$ is larger than the largest $J\left(R_{i j}\right)$.

b) Scaling laws. $/ 4 \%$ - In the low temperature range, only the interactions for $R>R(x)$ given by the equation (4) are important.

$\tilde{J}(R)=0$ for $R<R_{0} x^{-1 / 3}$;

$\tilde{J}(R)= \pm \frac{J_{0}}{\left(k_{F} R\right)^{3}}$ for $R>R_{0} x^{-1 / 3}$

This means that the value of $\tilde{J}_{0}$ (positive for exanple) which is the average of $J\left(R_{i j}\right)$ is irrelevant as long as the concentration is sufficiently small. When $x$ increases, one should find a transition from the spin-giass to an ordinary magnetic phase (ferromagnetic for example) for a given value $x_{0}$ of $x$ and this transition should be abrupt.

With the interactions (9), we get immediately scaling laws. One can introduce reduced quantities $\frac{T}{x}, \frac{H}{x}, \frac{M}{x}, \frac{C}{x} \ldots$ The behaviour should be uni- versal. From this, one deduces immediately that the critical temperature $\mathrm{T}_{\mathrm{SG}}$ (if there is a spin glass phase) is proportional to the concentration, different from $T_{p}$, but of the same magnitude. Also, one gets for the specific heat and the magnetization :

$\frac{C}{x}=f\left(\frac{T}{x}, \frac{H}{x}\right)$

$\frac{M}{x}=g\left(\frac{T}{x}, \frac{H}{x}\right)$

From (10), one can say, that if the specific heat is a linear function of $T$ (at low $T$ for example), then the coefficient $\gamma$ is independent of the concentration. From (11) if the susceptibility in low field at low $T$ is constant, it must be independent of the concentration.

The scaling laws are very well obeyed for the thermodynamical properties but also for the hysterisis effects (remanent magnetization for example) 16/. This is somewhat surprising in view of the approximations which have been made. This result seens to prove that the criterion $k_{F}^{-1} \approx a \gg R_{i j}$ is the good one to validate equation (9) for the effective interactions (by the way, this is to my knowledge the only direct proof of the decreasing behaviour of the oscillations as $\mathrm{R}^{-3}$ ).

It is possible to destroy the scaling laws in two directions.

1) At high temperatures, there are deviations from $\frac{\mathrm{x}}{\mathrm{T}}$ or $\frac{\mathrm{x}}{\mathrm{H}}$ as expected $/ 7 /$.

2) If the mean free $\lambda$ path is short (by alloying with non magnetic impurities), the exchange interactions are multipled by the factor $e^{-r / \lambda}$ and one should observe deviations from the scaling laws. This has been demonstrated in a series of experiments by Souletie / $8 /$.

The scaling laws seem to be a solid ground for real spin glasses but quantitative results should be obtained for the effective interactions $\tilde{J}(R)$.

c) Specific heat at low temperatures.- The linear behaviour of the specific heat at law temperatures was interpreted by a distribution $P\left(H_{m}\right)$ of molecular fields by various people $/ 4 /, / 9 /$. If $P(0)$ is different from zero, this explains the behaviour of $\mathrm{C}$. With an Ising model and no correlations between the spins, it is easy to show that this is the case. If the interactions are long-range so that many spins interact with a given spin, the distribution law $\mathrm{P}\left(\mathrm{H}_{\mathrm{m}}\right)$ is Gaussian in general (central 
limit theorem). However, with the effective interactions (9), the distribution is Lorentzian, as it can be easily proved. In order to obtain a constant $\gamma$ (with regards to the concentration $x$ ) one has to take the effective interactions as given by equations (9). This prescription kills the modulations in the wings of $\mathrm{P}\left(\mathrm{H}_{\mathrm{m}}\right)$ which would have appeared with the use of non-renormalized $J(R)$. These results are obtained neglecting the crystalline character of the alloy. More detailed calculations have been done by Klein and Brout /5/ ; they give essentially the same results.

This explanation seems perfect. But the interactions are not Ising but rather Heisenberg interactions and there is no reason to believe in anisotropy fields sufficiently strong to bring back the system to the Ising case. With Heisenberg interactions, $P\left(H_{m}\right)$ behaves as $H_{m}^{2}$ and this gives $C \propto x^{2} T^{3}$.

This situation has been a puzzle for years. Today a possible solution can be put forward. In ordinary glasses, the specific heat is also roughly linear. A model has been proposed by Anderson, Halperin and Varma /10/ in order to describe the glasses : it is based on the existence of two-level systems, which are characteristic of the complicated and non ergodic phase space of glassy systems. This model has been widely used to explain with success the properties of glasses. We may think that a similar model could be used for spin glasses. This idea is strong1y supported by the recent work of Villain /11/ : starting from a spin glass model with isotropic $x-y$ classical spins, Villain has shown that the model exhibits two-level systems for two spatial dimensions : it is equivalent to an Ising system, the two levels being related to the sense of orientation of the spin direction. Though the three dimensional mode1 /12/ woes not give similar results, the two dimensional case is a good support of the ideas of Anderson, Halperin and Varma. Such a mechanism may by true for real spin glasses in 3 dimensions and explain the linear behaviour of $\mathrm{C}$. Clearly, there is there a direction to solve this problem.

The linear behaviour of the specific heat is supported by the numerical simulations done by Walker and Waldstedt /13/. They have studied a classical Heisenberg Hamiltonian in three dimensions with real RKKY interactions. They determine the elementary excitations (extended and localized ones) and find after quantization a linear specific heat.
Before ending with this problem, it should be remarked that the experimental values of $\mathrm{C}$ are not sufficiently precise to forbid a linear behaviour in a wide range of temperatures but which would extrapolate to a finite temperature, with a small non $1 i-$ near regime near $T=0$. The same remark applies to the simulation of Walker and Waldstedt /13/.

A final solution of the specific heat problem (on the experimental and theoretical point of view) would certainly give a lot of informations on the spin glass state at low temperature.

d) Remark.- Until now we have discussed the real case of dilute magnetic alloys. The probability distribution of the exchange interactions depends upon the spatial configuration of the magnetic atoms which are randomly distributed. These systems are very inhomogeneous.

In many models of spin glasses, the spins are on a lattice and the exchange interactions are restricted to first neighbours with the same probability law. These models are much more homogeneous and quite far from real spin glasses (CuMn for example). This does not mean that these models have no interest (on the contrary as we shall see later) ; but one should be careful when extending the results of such models to real spin glasses.

3. HYSTERISIS, REMANENCE AND TRAINING.- The study of the magnetic properties of spin glasses at low temperatures is very rich and it is impossible in a short review to describe them in detail. Let us point out the main results and interpretations.

a) Remanent magnetizations. - The various remanent magnetizations which will be discussed below, have been known years ago /14-17, 19/, but the universality of the results and the fact that they obey scaling laws has been recognized later (Tholence /18/).

The thermoremanent magnetization (TRM) is obtained after cooling in a field from high temperature (above $\mathrm{T}_{\mathrm{SG}}$ ). The isothermal magnetization (IRM) is obtained when a field is applied then suppressed at the same temperature (well below $\mathrm{T}_{\mathrm{SG}}$ ). The two magnetizations saturate in high fields to the same value $\sigma(T)$. Typical curves are given on figure 2 (taken from reference $/ 6 /$ ).

The saturated magnetization $\sigma(T)$ obeys an exponential law :

$\sigma(T)=\sigma_{0} e^{-\alpha \frac{T}{x}}$ with $\sigma_{0}=a x$ 
This effect has been observed by Tournier years ago $/ 17 /$. The exponential behaviour of $\sigma$, in addition to the small values of a gives very small values of $\sigma(\mathrm{T})$ near $\mathrm{T}_{\mathrm{SG}}$. But, it is certain that $\sigma(\mathrm{T})=0$ above $\mathrm{T}_{\mathrm{SG}}$ and at $\mathrm{T}_{\mathrm{SG}}$. Formula (12) should be modified near $\mathrm{T}_{\mathrm{SG}}$; but the experiments are very difficult in this range of temperature.

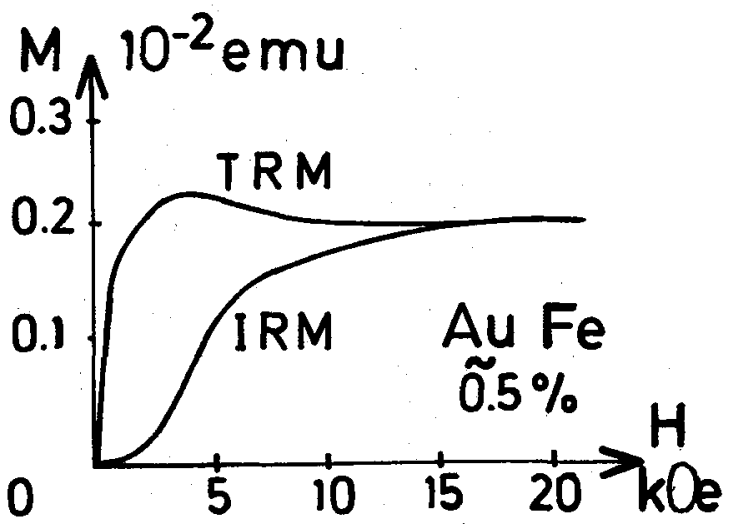

Fig. 2 : Field dependence of the thermoremanent magnetization (T.R.M.) after cooling to $T=1.2 \mathrm{~K}$ in a field $\mathrm{H}$ and field dependence of the isothermal remanent magnetization (I.R.M.) obtained when a field $\mathrm{H}$ applied at $1.2 \mathrm{~K}$ is suppressed.

The dynamics of the magnetization processes is shown on figure 3 : the magnetic alloy has been cooled well below $\mathrm{T}_{\mathrm{SG}}$ in zero magnetic field; at $t=0$, a magnetic field is applied until time $t_{0}$ (curve a). The magnetization is given by the curve (b). At $t=0$, the magnetization jumps in a yery small time from $A(M=0)$ to $B(M \neq 0)$; then it obeys a logarithmic law. When the field is turned off to zero, $M(t)$ jumps from $C$ to $D$ with $C D=B A$; then it has a logarithmic behaviour /20/. Detailed experiments on the time evolution of the remanent magnetization are reported by Prejean at this conference $/ 21 /$.

All the experiments which have been described above obey scaling laws : this observation proves that the remanence and its dynamics are properties of a good solid solution and that they are not the consequence of chemical clustering or other parasitic effects.

b) Interpretation within the model of small grains of Néel. - The magnetic behaviour of small ferro or antiferromagnetic grains (or domains) coupled by anisotropy forces has been explained by Néel /22/. This is called "superparamagnetism" : the grains (domains) have a magnetic moment and at high temperature they behave as paramagnets.

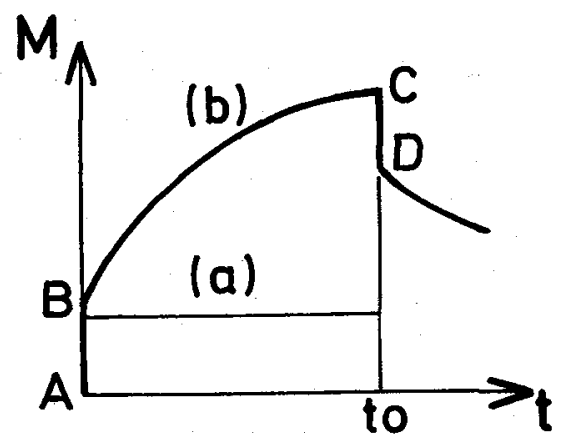

Fig. 3 : Dynamics of the magnetization processes. curve (a) Applied magnetic field curve (b) Magnetization

Below a blocking temperature $\mathrm{T}_{\mathrm{b}}$, the anisotropy energy gives rise to typical remanent effects : the TRM is larger than the IRM; in low fields the TMR. varies as $\mathrm{H}$, the IRM as $\mathrm{H}^{2}$; they have the same saturated value at each temperature. This theory relies on two main assumptions : 1) The existence of potential barriers of energy $W$ separating two easy orientations of the domain. The average time for the magnetization to jump over the barrier by thermal fluctuations is given by an Arrhenius law :

$\tau=\tau_{0} \frac{W}{e^{k} B^{T}}$

2) It is assumed that the distribution of $P(W)$ of the energy $W$ is constant over a wide range of energies. These assumptions resemble the two-level system of Anderson Halperin and Varma /10/ with the difference that in their case tunneliing effects replace the classical law (13).

The fact that the remanent effects of dilute magnetic alloys behave as in the theory of Nêe 1 has been recognized since a very long time $/ 14-17 /$. Let us sumarize how this theory is applied to spin glasses $/ 18,23 /$.

The dilute alloy behaves like an assembly of domains characterized by the number $n$ of spins which they contain and their magnetization $\mathrm{Mg}$. The anisotropy energy is due to the magnetic dipolar couplings between the spins (which decreasing as $\mathrm{R}^{-3}$ obeys the scaling laws). The domains have a distribution of activation energies $W$, which gives a distribution of blocking temperatures $T_{b}$. The magnetization Mg is taken as distributed by a Gaussian law with an average value 0 . 
With these assumptions, one explains the main results of the preceding section for the TRM and the IRM, the equation (12) for the saturated magnetization and the logarithmic variation of $M(t)$. The number $n_{0}$ of spins within a domain is independent of the concentration and proportional to the ratio of the amplitudes of the exchange interaction and the dipolar interaction; $\mathrm{n}_{\mathrm{o}}$ is approximately equal to 260 for CuMn alloys and to 500 for AuMn alloys.

This theory explains the main experimental results. Nevertheless, various questions arise: 1) on the importance of dipolar interactions. What would happen if they were strictly zero $\left(n_{0} \rightarrow \infty\right)$ ? 2) on the importance of the time scale of the measurements.

3) How the existence of a distribution of blocking temperatures $T_{b}$ can agree with the existence of $a$ well defined critical temperature $\mathrm{T}_{\mathrm{SG}}$ ?

These questions will come back later and are crucial.

c) Magnetization jumps and hysterisis loops.- A number of experiments on the hysterisis of dilute magnetic alloys show magnetization jumps at low temperatures. Evidences for this was first given by Tournier in AuFe and CuCo alloys /17,19/. Also, shifted hysterisis loops have been observed since a long time by Kouvel /15,24/ in CuMn and AgMn alloys.

The hysterisis cycles of CuMn alloys well below $\mathrm{T}_{\mathrm{SG}}$ have been measured and are reported at this conference $/ 25 /$. The hysterisis cycle is observed to be "square", when the measurement of rapid magnetization changes* is possible (figure 4). Characteristic features of the cycle are found : it is non symmetric with respect to zero field ; the complete cycle can be described by two reversible parts $(A B$ and $D F$ ) connected by two almost complete magnetization jumps. The interpretation is that during these jumps all magnetic moments reverse their signs. Sometimes, only $50 \%$ of the magnetization is reversed, giving a net magnetization near zero value : the interpretation is that there are now two domains with opposite directions of the magnetization.

Many questions are raised by these experiments about the domains, the existence of a threshold field $H_{c}$, and the dynamics of the magnetization. Here again, time scales are important and the results are not the same for CuMn alloys or for AuFe alloys.

4. CRITICAL TEMPERATURE AND SPIN GLASS PHASE.- The existence of a critical temperature above which a new phase, the spin glass phase appears is linked with the existence of a "cusp" in the susceptibility.

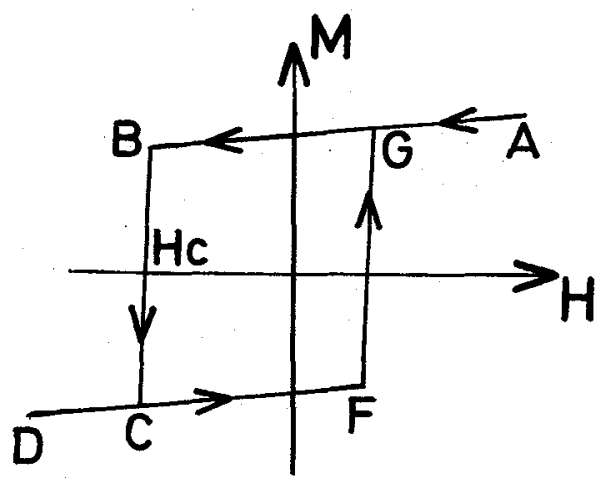

Fig. 4 : Hysterisis loop (schematic figure taken from reference $/ 22 /$ ).

a) Cusp of the susceptibility,- In 1972, Canella and Mydosh /26/ showed very clearly the existence of a cusp in the susceptibility. The cusp appears more evidently than in earlier experiments because the measurements were done in small fields modulated at low frequencies : this technique gives directly $\frac{\mathrm{d} M}{\mathrm{dH}}=\chi$. Earlier experiments were done in much larger fields, giving $M(H)$ and the cusp is less apparent in those conditions $/ 2,15,16,17 /$.

Figure 5 shows how the susceptibility is modified when the amplitude of the average field increases : the cusp disappears giving rise to a maximum which broadens with increasing field and the maximum is generally displaced towards lower temperatures.

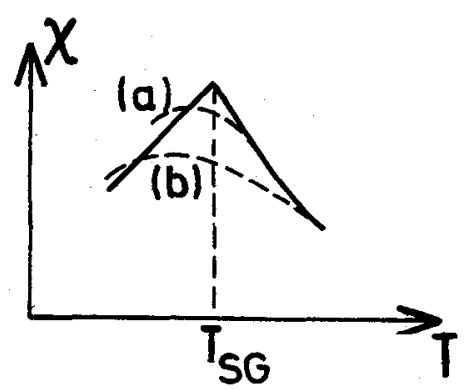

Fig. 5 : Typical behaviour of the susceptibility as raeasured in an alternative field - amplitude 0.1 gauss (a) 8 gauss (b) 16 gauss.

In those conditions, it is clear that one can define a critical temperature only in the limit $\mathrm{H} \rightarrow 0$. One can also define reversible and irreversible susceptibilities $/ 6,23 /$ as shown on figure 6 : the reversible susceptibility $\chi_{R}(T)$ is the instantaneous response to a small magnetic field (alternative measurements for example). The irreversible susceptibility $\chi_{I R}$ is an additive part which is 
observed at constant field after a long time delay (the scale of time depends on the temperature and on the concentration of the alloy).

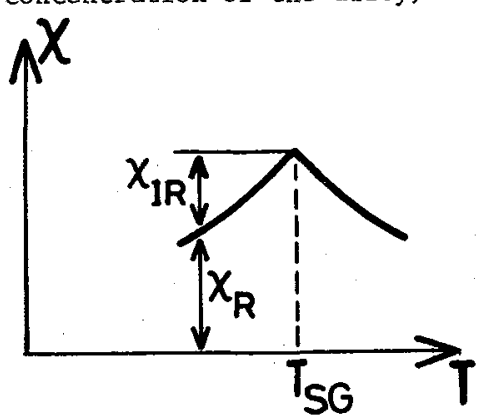

Fig. 6 : Reversible and irreversible susceptibility.

The total susceptibility $X_{T}=X_{R}+X_{I R}$ is independent of the temperature, at least near the temperature of the cusp.

Very recent experiments which are reported in this conference by Löhneysen, Tholence and Tournier /27/ show that the cusp depends upon the frequency of the alternative field. In their study of $\left(\mathrm{La}_{1-\mathrm{x}} \mathrm{Gd}_{\mathrm{x}}\right) \mathrm{Al}_{2}$ alloys $(\mathrm{x}=0.6 \%$ and $\mathrm{x}=1 \%)$, the frequencies varying from $0.02 \mathrm{~Hz}$ to $1140 \mathrm{~Hz}$, they find that the maximum of $X$ is displaced to lower temperatures when the frequency decreases, while the value of the maximum of $x$ increases.

There are also cases where the frequency dependence of $\mathrm{x}$ has not been observed, in Ag Mn for example (see the article of Dahlberg Hardiman and Souletie).

The frequency dependence of the cusp enlights the results of Mössbauer and neutron scattering studies of spinglasses which do show a "critica1" temperature which is different from that obtained through the observation of the cusp. In the neutrons experiments of Murani /28/, the "critical" temperature is 1arger by a factor 1.25 than the temperature of the cusp of the susceptibility. I shall not discuss these experiments in detail (see the article of Murani /29/ at this conference), but it appears now clearly that the conclusions deduced from various experiments have to take into account the time dependence (or frequency dependence) of themethods.

These last experiments show that the time dependent effects observed at low temperatures show up also near the "critical" temperature. This adds an argument to the fact that a "good" theory should be able to give an explanation of the time-dependent effects.

These experiments show also that the critical temperature $T_{c}$ has to be defined taking two Iimits : $H \rightarrow 0$ and $\omega \rightarrow 0$. The critical temperature is very easily hidden, and its definition is far from the definition of the critical temperature of an ordinary second order transition. It is a subtile transition, but its existence seens well established. The existence of this phase transition does not seem to be linked with a singular behaviour of the specific heat $C$ at $T_{C}$ (there is absolutely no experimental evidence of a singularity of $\mathrm{C}$ ) in contradiction with ordinary second order phase transitions (ferromagnetic case for example).

b) The spin glass phase.- The existence of a sharp phase transition is based on two qualitative ideas. The first one is the following : in a second order phase transition the coherence length $\xi \rightarrow 0$ when $\left(T-T_{C}\right) \rightarrow 0$ and this remains true for a disordered medium. The scale of the disorder (here the statistical fluctuations of concentration) are always small compared to $\xi$ near $T_{c}$. The specimen looks "homogeneous" near $T_{c}$, with one critical temperature (and not a smooth distribution of critical temperatures). The same argument applies to other cases where disorder is fundamentally present : the percolation problem or the localization of Anderson.

The second idea is that the fluctuations of concentration gives rise to a phenomena of percolation : when $\mathrm{T}$ decreases below $\mathrm{T}_{c}$ "ordered" regions have a size $\xi$ which is infinite. This does not imply that the "disorder" is perfect above $\mathrm{T}_{c}$. In fact, it is better to speak of "islands" of local "order" for $T>T_{c}$ and "lakes" of "disorder" for $\mathrm{T}<\mathrm{T}_{\mathrm{c}}$ (figure 7).

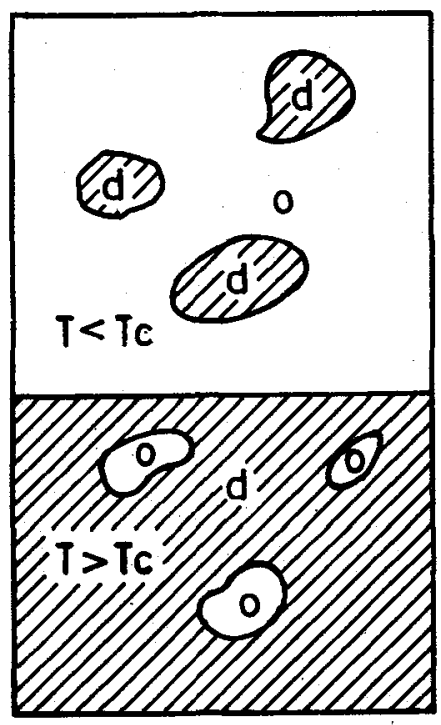

Fig. 7 : Qualitative description of a spin glass above and below $T_{c}$. 
Here also, one should pay attention to the word "order" which does not mean usual long range order with an order parameter as in usual second order: phase transitions. Here, this means some kind of cooperative behaviour which extends at short distances above $\mathrm{T}_{\mathrm{c}}$ and long distances below $\mathrm{T}_{\mathrm{c}}$. These qualitative ideas are basic in the phenomenological approaches of Adkins and Rivier /30/ and of Smith /31/ ("mictomagnetism").

One may ask more about the nature of the spin glass state. The phase transition occurs without broken symmetry, without the appearance of a long range order parameter in the low temperature phase. In ordinary phase transitions, the system is non exgodic below $\mathrm{T}_{c}$ : only part of the phase space is available. Here, in this random system, non ergodicity is fundamental ; the phase space must be very complicated with a lot of yalleys (ground states) separated by very high passes. This description is in qualitative agreement with the existence of the subtle hysterisis phenomena which have been described in the paragraph 3 , with the importance of the time in all experiments and with the difference in the results which are obtained when cooling under different magnetic fields. The entropy of the spin glass should be very large and it is possible to have a finite entropy at zero temperatures in the thermodynamical limit. This idea is supported by the experimental observation that the total entropy between $T=0$ and $T=T_{c}$ is a rather small fraction of the total entropy available. Measurements of $\mathrm{C}$ at high temperature could give an answer to this question of finite entropy at $\mathrm{T}=0$. (For a discussion of non ergodicity in random systems see Anderson /32/).

It appears that the spin glass phase and the spin glass phase transition are quite peculiar and new. This will show up very clearly in the next section.

5. MEAN FIELD THEORY I.- In the historical study of phase transitions, the first steps have always been to define a "mean field" theory, then a Ginzburg Landau functional energy, then fluctuations, then renormalization.

Let us confine ourselves to the problem of defining a mean field theory and in this whole section, we shall restrict the discussion to Ising spins though initia1ly, the Edwards and Anderson theory was describing the classical Heisenberg problem.
Consider the Hamiltonian (Anderson /32/)

$\mathrm{H}=-\sum_{(i j)} \mathrm{J}_{i j} \mathrm{~s}_{i} \mathrm{~s}_{j} \quad$ with $s_{i}= \pm 1$

and a given probability distribution of the exchange parameters $J_{i j}$ : In the ordinary mean field theory ("molecular" field), one would come back to a one spin problem and write self-consistent equations : $\left\langle S_{i}\right\rangle=\operatorname{th}\left(\beta \sum_{j} J_{i j}\left\langle S_{j}\right\rangle\right)$

If there is a critical temperature, one can linearize (15) which gives :

$\left.\mathrm{k}_{B} \mathrm{~T}<\mathrm{S}_{i}\right\rangle=\sum_{j} \mathrm{~J}_{i j}<\mathrm{S}_{j}>$

The critical temperature is usually given by the highest eigenvalue of equation (15). With random $J_{i j}$, this is no more true. The eigenvalues of (16) can have eigenvectors which correspond to $10 \mathrm{calized}$ states (involving essentially a finite number of spins) or extended states (involving all the spins with a comparable weight for each spin). From what we know about localization, the localized states will correspond to the highest eigenvalues of (16) and their will be sharp transition to extended states. $k_{B} T_{C}$ should be equal to the highest extended value $\lambda_{0}$ of the eigenvalues $\lambda$.

$\underset{\text { eigenvectors }}{\text { extended }} \lambda_{0}$

This qualitative description is in agreement with the ideas of section II and the same consequence emerges : the initial $J_{i j}$ have to be "renormalized". It is in agreement with the simulation of Walker and Waldstedt /13/ who do find localized and extended elementary excitations.

Two comments about this discussion :

1) depending on the dimensionality $\lambda_{0}$ may be $>0$ or be pushed to zero value. In that case, this defines the lower critical dimensionality $d_{c}^{m i n}$ (see section 8 ).

2) $\lambda_{0}$ should be highly degenerate in order to find in the spin glass phase the large number of "equivalent" valleys in phase space discussed in section $4 \mathrm{~b}$ and which are characteristic of the non ergodic behaviour of random systems.

The conclusion of this discussion is that the "ordinary" mean field theory cannot be used and equation (15) has to be modified.

a) Theory of Edwards and Anderson /33/.- The fundamental new concept of Edwards and Anderson is to an introduce the quantity : 
$q\left(t_{1} t_{2}\right)=\overline{\left\langle S_{i}\left(t_{1}\right) s_{i}\left(t_{2}\right)\right\rangle}$

where $\langle\ldots\rangle$ means thermal average and $\ldots$, average over the spins. In the spin glass phase, $q\left(t_{1} t_{2}\right)$ should behave as :

$\left|\lim _{1}-t_{2}\right|+\infty \quad q\left(t_{1}, t_{2}\right)=q>0$

Wherease it is zero above $\mathrm{T}_{\mathrm{c}}{ }$

Equation (18) introduces a kind of "memory" effect, which describes globally the limitations of the time fluctuations of a given spin. $q$ is in this theory the parameter of interest, though it is very different from usual order parameters which are linked with broken symmetry and with long range order in space. $q$ is a parameter which is linked with some kind of "order" in time.

To formulate a thermodynamical description of the system, let us derive the $\mathrm{E}$ A method in the Ising case.

Consider an Ising model with distributions of $\mathrm{J}_{i j}$ (Sherrington and Kirkpatrick /34/) :

$P\left(J_{i j}\right)=\frac{1}{(2 \pi)^{1 / 2} J} e^{-\frac{\left(J_{i j}-J_{o}\right)^{2}}{2 J^{2}}}$

Each spin interacts with $z$ spins, $z$ being of the order of the total number of spins (in ordinary phase transitions, the mean field theory is exact in this 1imit).

$J_{0}$ and $J$ are scaled as $J_{0}=\frac{\tilde{J}}{z}, J=\frac{\tilde{J}}{I / 2}$ so that both $\tilde{J}_{0}$ and $\tilde{J}$ are intensitive quantities.

In order to calculate the free energy one has to take the average of Log $\mathrm{z}$ ( $\mathrm{z}$ being the partition function) because the system is quenched (averaging $Z$ is valid for annealed system, that is, in our case, mobile interactions). Averaging $\log \mathrm{z}$ is difficult but if one writes :

$\log z=\lim _{n \rightarrow 0} \frac{1}{n}\left(z^{n}-1\right)$

the problem is reduced to the average value of $z^{\mathfrak{n}}$. This can be done with the introduction of replicas : n strictly identical systems characterized by spins $\mathrm{s}_{i}^{\alpha}$. The problem is reduced to the calculation of $\mathrm{Z}^{\mathrm{I}} \mathrm{z}^{2} \ldots \mathrm{z}^{\mathrm{n}}$ (n being an integer) and the analytic continuation to $\mathrm{n} \rightarrow 0$. The introduction of replicas and their interpretation is somewhat delicate : two replicas $\alpha$ and $\beta$ can be understood as the same system at two times $t_{1}$ and $t_{2}$ with $\left|t_{1}-t_{2}\right| \rightarrow \infty$.

Now how one can average with the distribution law /19/ which gives : $\mathrm{z}^{\mathrm{n}}=\mathrm{e}^{-\mathrm{H}_{\text {eff }}}$ where :

$$
\begin{aligned}
& -\beta \quad H_{\text {eff }}=\frac{\beta^{2} J^{2}}{4} \sum_{\substack{\alpha, \beta \\
i \neq j}} s_{i}^{\alpha} s_{j}^{\alpha} s_{i}^{\beta} s_{j}^{\beta}+\sum_{\substack{\alpha \\
i \neq j}}^{\beta J_{o}} \frac{J_{j}}{2} s_{i}^{\alpha} s_{j}^{\alpha}+ \\
& \beta \mathrm{h} \sum \mathrm{s}_{i}^{\alpha} \\
& i \neq j
\end{aligned}
$$

where the magnetic field $h$ is uniform and the sum on all replicas.

The E A method is then a classical mean field theory ; one defines

$$
\begin{aligned}
& \left\langle\overline{s_{i}^{\alpha}>}=n\right. \\
& \left\langle\overline{s_{i}^{\alpha} s_{j}^{\beta}>}=\delta_{i j}\left(\delta_{\alpha \beta}+\left(1-\delta_{\alpha \beta}\right) q\right)\right.
\end{aligned}
$$

which means that there is no correlation between sites $i$ and $j$, but a correlation between the spin $(i, \alpha)$ and the spin $(i, \beta)$ with the same $i$. Thus $q$ should be the same as defined in equation (18)

The fundamental belief is that :

$\mathrm{m} \rightarrow 0$ when $\mathrm{h} \rightarrow 0$ at all temperatures

$\mathrm{q} \rightarrow 0$ when $\mathrm{h} \rightarrow 0$ when $\mathrm{T}>\mathrm{T}_{\mathrm{c}}$

$\mathrm{q} \neq 0$ when $\mathrm{h} \rightarrow 0$ when $\mathrm{T}<\mathrm{T}_{\mathrm{c}}$ (the spin glass phase) Usual factorization of (21) brings back the problem to a one-spin problem which can be solved. After anaiytic continuation and extraction of the linear term in $n$, one gets the final result :

$$
\begin{gathered}
F=-k_{B} T\left(\frac{\beta^{2} \tilde{J}^{2}}{4}(1-q)^{2}+\frac{1}{(2 \pi)} \int_{-\infty}^{+\infty} e^{-\frac{x^{2}}{2}} d x\right. \\
\log \left(2 \operatorname{ch}\left(\beta \tilde{J} q^{1 / 2} x+\beta \tilde{J}_{0}^{1 / 2}+\beta h\right)\right\}
\end{gathered}
$$

$F(q, m)$ is a variational function with 2 parameters. Finding the extrema of $F(m, q)$ gives $\frac{\partial F}{\partial m}=0=\frac{\partial F}{\partial q}$ and one obtains :

$\mathrm{m}=\frac{1}{(2 \pi)^{1 / 2}} \int_{-\infty}^{+\infty} e^{-\frac{x^{2}}{2}} d x \operatorname{th}\left(\beta \tilde{J q}^{1 / 2} x+\beta \stackrel{J}{J}_{0}+\beta h\right)$

$q=\frac{1}{(2 \pi)^{1 / 2}} \int_{-\infty}^{+\infty} e^{-\frac{x^{2}}{2}} d x \operatorname{th}^{2}\left(\beta \tilde{J}^{1 / 2} x+\beta \tilde{J}_{0}+\beta h\right)$

The discussion of equations (24) (25) and (26) gives the main following results :

1) There is always a phase transition : at $k_{B} T_{c}=\tilde{J}_{0}$ if $\tilde{J}_{o}>\tilde{J}$ and the low temperature phase is ferromagnetic ; when $\tilde{J}_{0}<\tilde{J}$ the phase transition occurs for $\mathrm{kT}_{c}=\tilde{\mathrm{J}}$ and the low temperature phase has the expected spin glass properties as discussed above.

Thus, it appears that $\tilde{J}_{0}$ is "irrelevant" as long as it is smaller than $\tilde{J}$; it leads only to a modification of the susceptibility : 


$$
x\left(\tilde{J}_{0}\right)=\frac{x\left(\tilde{J}_{0}=0\right)}{1-\tilde{J}_{0} \times\left(\tilde{J}_{0}=0\right)}
$$

2) The properties of the system near the transition temperature for the spin glass case $\left(\widetilde{J}_{0}<\tilde{J}\right)$ are :

The susceptibility $\chi(T)$ shows a cusp for $T=T_{c}$ and obeys the law of Fisher $X=B(1-q)$.

The specific heat shows also a cusp, though it is slightly less apparent $\left(\frac{\mathrm{dC}}{\mathrm{dT}}=0\right.$ when $\left.\mathrm{T} \rightarrow \mathrm{T}_{\mathrm{c}}^{-}\right)$

The parameter $\mathrm{q}$ behaves as $\frac{\mathrm{T}_{\mathrm{c}}-\mathrm{T}}{\mathrm{T}_{\mathrm{c}}}$ near $\mathrm{T}_{\mathrm{c}}$.

3) Low temperature properties which shall be discussed later.

Edwards and Anderson were diseussing the classical Heisenberg case. Extensions to the classical $n$ vector model is trivial. The extension to the quantum Heisenberg case though slightly more subtle has been done by Fischer /35/.

b) Direct derivation /36/.- A direct deriyation of the results of 5 a) can be done without the replica method. I did not published it in 1975, because there was no need to do it, but if clarifies the difficulties which have appeared later.

With the same Hamiltonian, for an Ising system and the probability $P\left(J_{i j}\right)$ of equation (19) one can calculate the distribution $P(\zeta)$ of the molecular field for the spin $i$ :

$P(\xi)=\underset{(i j)}{I I} \int \cdots \int P\left(J_{i j}\right) d J_{i j} \delta\left(\zeta-\sum_{j} J_{i j}<s_{j}>-h\right)$

The following assumptions are made :

1) $\overline{\left\langle s_{j}\right\rangle}=m ; \overline{\left\langle s_{i}\right\rangle\left\langle s_{j}\right\rangle}=q \delta_{i j}$

2) Each spin interacts with many spins ( $Z$ of the order $N$ ).

3) There is no correlation between $J_{i j}$ and $\left\langle s_{j}\right\rangle$. so that one can take the averages independently.

Taking the Fourier transform of (29) and making use of the preceding assumptions, one finds

$$
P(\zeta)=\frac{1}{(2 \pi)^{1 / 2} \Delta^{1 / 2}} e^{-\frac{\left(\zeta-m \tilde{J}_{0}-h\right)^{2}}{2 \Delta^{2}}}
$$

where $\Delta=\tilde{J} \quad q^{1 / 2}$

Writing self-consistent equations for $m$ and $q$ : $m=\int_{-\infty}^{+\infty} P(\zeta)$ th $\beta \zeta \quad d \zeta$ $q=\int_{-\infty}^{+\infty} P(\zeta) \operatorname{th}^{2} \beta \zeta d \zeta$

gives back the results of the $\mathrm{E}$ A method, equations (25) and (26). This does not determine $F$; but if we look for a free energy which gives the good ferromagnetic limit when $\tilde{J}=0$, then $F(m, q)$ is uniquely determined and is given by equation (24).

In this derivation, one sees very well that the field $\xi$ is calculated at site $i$ as if that site $i$ and a given site $j$ were completely uncorrelated. In fact there exists such a correlation and a feedback from $i$ to $j$ which forbids the hypothesis of non correlation. We shall come back on this point.

The same direct method can be used for classical $n$ vector spins and they give the same results than the E A method. On the contrary, this is not the case for quantum spins and this direct derivation does not give the results of Fischer /35/. (Why ?)

c) The "solvable" model of Sherrington and Kirkpatrick /34/ and the difficulties.-

In usual second order phase transition, one knows that the mean field theory is valid when the number $z$ of interacting neighbours is of the order the total number $\mathrm{N}$ of the spins. This was the starting idea of Scherrington and Kirkpatrick. They took the distribution (21) for the exchange constants and made an exact calculation of the free energy. The only problem, in that derivation, is that they interverted the $\lim n \rightarrow 0$ and the thermodynamical limit $\mathrm{N} \rightarrow \infty$, in order to make a usual steepest descent (or saddle point) integration. The good order of the limits should be : $\lim _{N \rightarrow \infty} \lim _{n \rightarrow 0} \phi$ and not $\lim _{n \rightarrow 0} \lim _{N \rightarrow \infty} \phi$ as they did.

This fact was not apparent in the derivation of 5a) because after the mean field factorization of $\mathrm{H}_{\text {eff }}$, the good order of the limits could be kept. Now, the difficulties began :

1) Sherrington and Kirkpatrick remarked that the entropy at $T=0$ was negative. A positive entropy would not be a shocking result, as it has been discussed in section 4 but a negative entropy is shocking. Negative entropy appeared also for the classical Heisenberg case, but we know that with continuous variables, one gets dificulties with the entropy at low temperatures. The remedy is quantization, that is discretization of the energy levels. What is shocking here, is that we start from a discrete Ising 
Hamiltonian*

2) At least as important is the remark that the variational function $F(q)$ is a maximum when $T>T_{c}$ for $q=0$ and when $T<T_{c}$ for $q=q_{0}=\frac{T_{c}-T}{T_{c}}$. Figure 8 shows the case when $\mathfrak{J}_{0}=0$ and $h=0$. This shows that the solution $q \neq 0$ below $T_{c}$ is above the analytic continuation of the high temperature free energy $F(q=0)$ (see Figure 9 ).

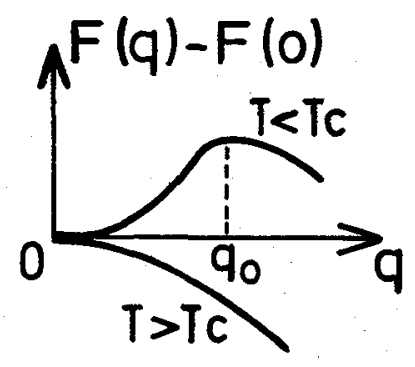

Fig. 8 : The free energy $F$ as a function of the variational parameter $q$ above and below $T_{c}$.

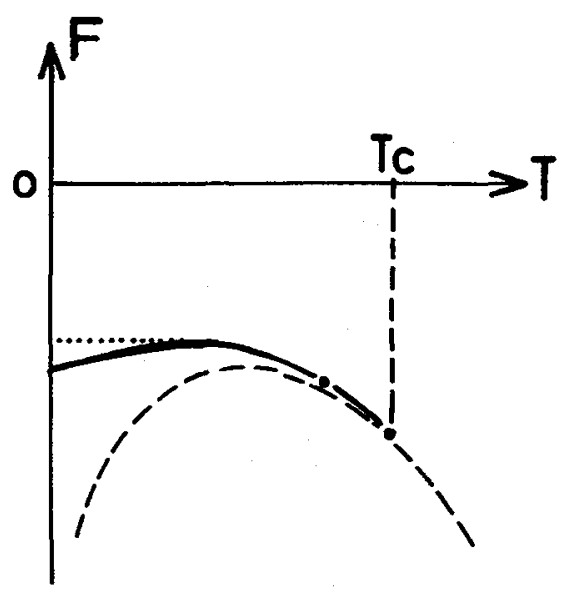

Fig. 9: The free energy as a function of temperature of the "solvable" mode1 of Sherrington and Kirkpatrick- - $\mathrm{q}=0-\mathrm{q} \neq 0 \ldots$ TAP

The figure 10 shows the difference between an ordinary phase transition : $F(T, q)-F(T, q=0)$ is negative (a) and varies as $\left(T_{c}-T\right)^{2}$ for an ordinary phase transition. For the spin glass case, it is positive (b) and varies as $\left(\mathrm{T}_{\mathrm{c}}-\mathrm{T}\right)^{3}$.

In ordinary phase transitions, one neglects $F(T, q=0)$ which has no importance. Here on the

* A spherical model of spin glasses has been studied $/ 37 /$, without using the replica method and it can be solved exactly. But the ordinary spherical model gives a negative entropy because the discretization $\left(S_{i}= \pm 1\right)$ is relaxed and the spin variables become continuous with a global constraint. The solution /37/ (though interesting) does not bring any light on the problem of negative entropies. contrary it has to be taken into account : without that term, the specific heat below $T_{c}$ would be negative.

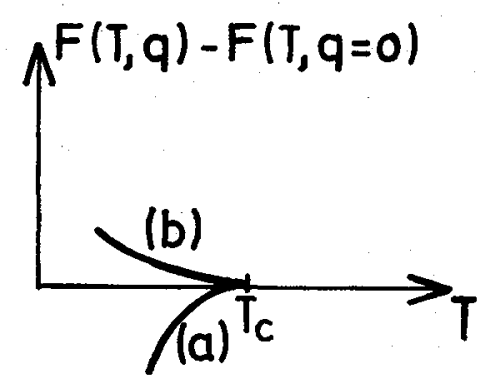

Fig. 10: The different behaviours of the free energy (a) ordinary second order transition

(b) spin glass phase transition

In conclusion of this section, we can say that the $E$ A method introduces a new type of phase transition, with a very different behaviour than in ordinary second order phase transitions. It brings also difficulties, which appear at the level of a mean field theory and which are unusual.

6. MEAN FIELD THEORY II. - The puzzling features of the E A theory have led various people to treat the problem from different points of view. The interest has been focused on the Ising case with $\tilde{J}_{0}=0$

a) The answer of Thouless Anderson and Palmer /38/.Avoiding the replica method, TAP study first the high temperature behaviour making a high temperature series expansion and find for the free energy per spin :

$F=-k_{B} T \log 2-\frac{\tilde{J}}{4 k_{B} T}-\frac{k_{B} T}{4 Z} \log \left(1-B^{2} \mathfrak{J}^{2}\right)$

When $k_{B} T>\tilde{J}$, the last term disappears $(Z \rightarrow \infty$ with $\mathrm{N}$ in the thermodynamical $1 \mathrm{mi}$ (). But this term diverges at $k_{B} T_{C}=\mathcal{H}$ and cannot be neglected ; it is a positive term in contrast with ordinary mean field theory as discussed in $5 \mathrm{c}$ ). This is a discrete signal of the occurence of a transition, nearly as discrete as the experimental one. Though no detailed calculations of the following terms in the expression (33) have been made, it seems most like1y that there is a transition and that it occurs for $\left.k_{B} T_{c}=\right\}$.

In order to obtain a mean field theory below $T_{c}$ without divergent terms TAP introduce a soluble mean field Hamiltonian $\mathrm{H}_{\mathrm{O}}$ and treat $\left(\mathrm{H}-\mathrm{H}_{\mathrm{O}}\right)$ as a perturbation. The convergence of the perturbation series (at least for the $\mathrm{z}^{-1}$ term) gives a constraint, which is, near $\mathrm{T}_{c}$ :

$q \geqslant \frac{T_{c}-T}{T_{c}}$ 
The mean field equations (15) are not valid and have to be replaced by

$\mathrm{m}_{i}=\operatorname{th\beta }\left(\sum_{j} \mathrm{~m}_{j} \mathrm{~J}_{i j}-\mathrm{m}_{i} \beta \sum_{j} \mathrm{~J}_{i j}^{2}\left(1-\mathrm{m}_{j}^{2}\right)\right)$

The second term in (35) is the response of the site $j$ to the mean value $m_{i}$ at the site $i$ : it must be removed from $m_{j}$ when one computes $m_{i}$. This is the kind of feed-back term which was missing in the $E A$ calculation as discussed in section $5 \mathrm{~b}$ ).

The corresponding free energy can be calculated for a given realization of the exchange cons$\operatorname{tants} \mathrm{J}_{\mathrm{ij}}$ :

$F=-\sum_{(i j)} J_{i j} m_{i} m_{j}-\frac{\beta}{2} \sum_{(i j)} J_{i j}^{2}\left(1-m_{i}{ }^{2}\right)\left(1-m_{j}{ }^{2}\right)$

$+\frac{k_{B} T}{2}\left(\left(1+m_{i}\right) \log \left(\frac{1+m_{i}}{2}\right)+\left(1-m_{i}\right) \log \left(\frac{1-m_{i}}{2}\right)\right)$

where the first term is the internal energy of the frozen lattice; the second gives the correlation energy of the fluctuations which are smaller by a factor $\left(1-m_{i}{ }^{2}\right)$ for each spin as compared to the high temperature case. The third term is the entropy of Ising spins constrained to mean values $\mathrm{m}_{i}$. Thus, it appears below $T_{c}$ a "blocking" effect on the spin fluctuations.

From equations (35) and (36), TAP derive the low temperature properties of the model : The ground state energy is slightly above the EASK result.

The entropy is zero at $T=0$; the specific yaries as $\mathrm{T}^{2}$ and the susceptibility as $\mathrm{T}$ (instead of $\mathrm{T}$ and constant respectively in the first approach of mean field). The distribution $P(\xi)$ of molecular fields starts from 0 linearly $P(\xi)=a|\xi|$ instead of a constant in the gaussian equation $(29)^{\mathrm{z}}$.

Near $T_{c}$, TAP find that the first and second derivatives of $F(q)$ with respect to $q$ vanish for : $\mathrm{q}_{0}=\frac{\mathrm{T}_{\mathrm{c}}-\mathrm{T}}{\mathrm{T}_{\mathrm{c}}}$ giving the "saddle" point configuration of figure 11 for $F(q)$. The constraint (34) forbids the region $\mathrm{q}<\mathrm{q}_{\mathrm{o}}$. TAP add that they "suspect that the free energy $F$ has the saddle point form sketched on figure 11 for all temperatures below $\mathrm{T}_{c}$, thus giving a line of critical points". Near $\mathrm{T}_{\mathrm{c}}$, the TAP solution gives back the results of Sherrington and Kirkpatrick /34/.

*

This low temperature behaviour is in quantitative agreement with recent numerical work of Kirkpatrick and sherrington /39/. The low temperature properties cannot be compared with experiments in real systems, the starting mean field Hamiltonian having nothing to do with the real one.

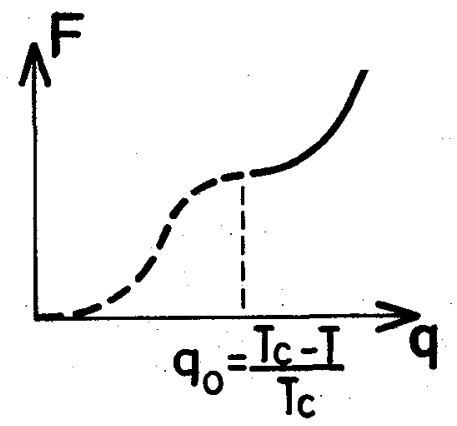

Fig. 11 : The free energy as a function of $q$, below $\mathrm{T}_{\mathrm{C}}$, as given by TAP.

b) Attempt towards a "Landau" model of the spin glass transition /40/. - A completely different approach gives results near $T_{c}$ which are nearly similar ("saddle" point configuration) (Blandin Gabay and Gare1 /40/).

Let us consider first two identical replica (same values of $\mathrm{J}_{i j}$ ) in order to define the parameter $q$. The Hamiltonians are

$H(s)=-\sum_{(i j)} J_{i j} s s_{i} s_{j}$
$H(\sigma)=\sum_{(i j)} J_{i j} \sigma_{i} \sigma_{j}$

$q$ can be defined as in reference /34/ as :

$q=<s \sigma>=\lim _{\mathrm{K} \rightarrow 0} \lim _{\mathrm{N} \rightarrow \infty} \frac{1}{N} \frac{\partial}{\partial K} \overline{\operatorname{LogTr} e^{\beta H(s)+\beta H(\sigma)+K \sum_{i} \sigma_{i}}}$

In this definition, one has to specify the sign of $\mathrm{K}$ (as in usual phase transitions) and $\mathrm{q}$ will be positive or negative depending upon the sign of $\mathrm{K}$ : one can have parallel or antiparallel replicas and more genera11y, for xy or Heisenberg classical models, two replicas can make an angle $\phi$ as shown on figure 12 .

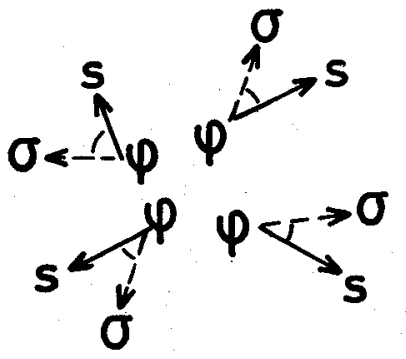

Fig. 12 : Two identical replicas : the replica $\sigma$ is obtained by a uniform rotation $\phi$ from the replica $s$. In equation (39) the thermodynamical limit $(\mathrm{N} \rightarrow \infty)$ has to be taken before the 1 imit $\mathrm{K} \rightarrow 0$; otherwise $q$ would be zero (same prescription than for ordinary second order phase transitions). An alternative definition of $q$ can be made with two real magnetic 
fields $h_{s}$ and $h_{\sigma}$, a double derivation with respect to $h_{s}$ and $h_{\sigma}$ and two limits $h_{s} \rightarrow 0$ and $h_{\sigma} \rightarrow 0$.

This shows clearly that $q$ has the characteristics of a second-rank tensor $\overline{\bar{q}}$.

The physical quantity q being defined with two replicas, let us replicate $m$ times these two replicas in order to get $\mathrm{rid}$ of the $\mathrm{Log}$ in equation (39). The $2 \mathrm{~m}$ replicas are coupled and we are linked to define the following parameters :

$$
\begin{array}{ll}
\mathrm{q}=\overline{\left\langle\mathrm{s}_{\alpha} \sigma_{\alpha}\right\rangle} \\
\left.\mathrm{p}=\overline{\left\langle\mathrm{s}_{\sigma} s_{\beta}\right.}\right\rangle=\overline{\left\langle\sigma_{\alpha} \sigma_{\beta}\right\rangle} \quad \alpha \neq \beta \\
\ell=\left\langle\overline{\mathrm{s}_{\alpha} \sigma_{\beta}}\right\rangle & \alpha \neq \beta
\end{array}
$$

where $\alpha$ and $\beta$ take the values $1 \ldots \mathrm{m}$.

$q$ is the physical parameter and we shall call $p$ and $\ell$ "unphysical" parameters. The symmetry gives :

$p>0, \ell$ and $q$ having the same sign as the sign of $K$ in equation (38)

If we make the assumption of the existence of the critical temperature, the symmetry for $T>T_{c}$ is described by the group of permutations $S_{2 m}$. The low temperature phase has on the contrary the symmetry $\mathrm{S}_{\mathrm{m}} \bowtie \mathrm{S}_{2}$. Then the spin phase transition appears in this case at the analytic continuation $(m \rightarrow 0)$ of the broken symmetry $\left(\mathrm{S}_{2 \mathrm{~m}} \rightarrow \mathrm{S}_{\mathrm{m}} \mathrm{S}_{2}\right)$ of a system of $2 \mathrm{~m}$ replicas.

Let us now construct the free energy $\mathrm{F}$ in analogy with the Landau theory. We make a development of $F$ near $T_{c}$ in function of the parameters $q, p$ and $\ell$. We suppose that the second order terms are proportional to $\left(T-T_{C}\right.$ ) and the third order terms to a constant $W$. Figure 13 gives the diagrams of order 2 $\left(\mathrm{q}^{2}, \mathrm{p}^{2}\right.$ and $\left.\ell^{2}\right)$ and $3\left(\mathrm{qp} \ell, \mathrm{p} \ell^{2}\right.$ and $\left.\mathrm{p}^{3}\right)$. The structure of these terms is imposed by the symmetry (eyen number of line for each point). Simple combinatorial analysis give :

$$
\begin{aligned}
F= & F_{0}+\lim _{m \rightarrow 0} \frac{1}{m}\left\{k_{B}\left(T-T_{c}\right)\left(m^{2}+(m-1) p^{2}+(m-1) l^{2}\right\}\right. \\
& \left.-\frac{W}{2}\left(2 m(m-1) q p l+m(m-1)(m-2) p l^{2} \div \frac{m(m-1)(m-2)}{3} p^{3}\right)\right\}
\end{aligned}
$$

Taking the limit $\mathrm{m} \rightarrow 0$ gives $\mathrm{F}=\mathrm{F}_{\mathrm{o}}+\mathrm{k}_{\mathrm{B}}\left(\mathrm{T}-\mathrm{T}_{\mathrm{c}}\right)\left(\mathrm{q}^{2}-\mathrm{p}^{2}-\ell^{2}\right)+\mathrm{W}\left(\mathrm{qp} \ell-\mathrm{p} \ell^{2}-\frac{\mathrm{p}^{3}}{3}\right)(42)$ The numerical constants for the second order and third order terms have been chosen so as to give back the $S K$ result when $q=p=\ell$. Thus $W=k_{B} T_{c}$. Equation (42) is the central result of this approach. The idea is now to eliminate the "unphysical" parameters $\mathrm{p}$ and $l$, the prescription being that $F$ should be an extrenum as regards to the parameters: $\frac{\partial F}{\partial p}=0 \quad \frac{\partial F}{\partial l}=0$

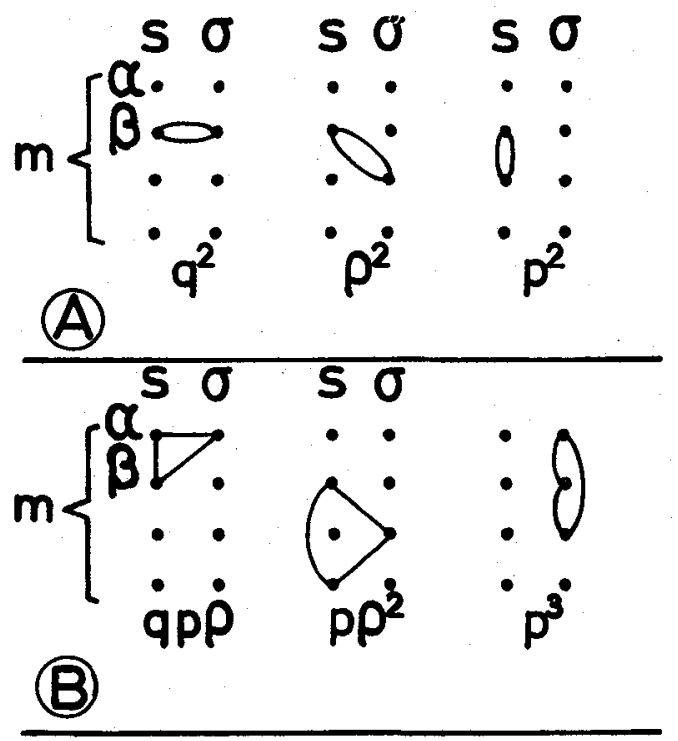

Fig. 13 : Construction of the free energy near $T_{c}$ A : second order terms $B$ : third order terms.

The discussion of equations (43) is somewhat long and we shall give only the results. These exists three solutions

1) $p=\ell=0$, we identify this solution with the high temperature phase and the free energy $F$ is : $F=F_{0}+k_{B}\left(T-T_{C}\right) q^{2}+4$ th order terms

2) $p=\ell=\frac{q}{2}+\frac{T_{c}-T}{2 W}$ which gives for $F(q)$

$F(q)=F(0)+k_{B} \frac{\left(T_{c}-T\right)^{3}}{6 W^{2}}+\frac{W}{12}\left(q-\frac{T_{C}-T}{W}\right)^{3}$

This is the low temperature phase with $K>0 \mathrm{q}>0$

$\ell>0$
3) $p=-\ell=-\frac{q}{2}+\frac{T_{c}-T}{2 W}$ which gives for $F(q)$

$\mathrm{F}(\mathrm{q})=\mathrm{F}(0)+\mathrm{k}_{\mathrm{B}} \frac{\left(\mathrm{T}_{c}-\mathrm{T}\right)^{3}}{6 \mathrm{~W}^{2}}+\frac{\mathrm{W}}{12}\left(\mathrm{q}+\frac{\mathrm{T}_{\mathrm{c}}-\mathrm{T}}{\mathrm{W}}\right)^{3}$

This is the low temperature phase with $\mathrm{K}<0 \mathrm{q}<0$ $\ell<0$

(45) and (46) exhibit very clearly the "sadd1e" point configuration as shown on figure 14. The free energy below $\mathrm{T}_{\mathrm{c}}(45)$ (or (46)) is not the analytic continuation of the free energy above $T_{c}$ (44). A1so the two branches (45) and (46) are not analytic and at $q=.0$ there exists a kink. The properties near $x_{c}$ for $C, X, S$ are the same than in the SK solution.

A question arises about the "saddle" point : does it remain in the following orders ? The discussion of the fourth order terms is long and we shall 
quote only the results $/ 40 /$ : whatever are the fourth order constants (they are 4), the "saddle" point gives rise to a maximum and a minimum. The minimum is the SK point. The free energy at the maximum is higher than the SK result. In this approach it appears that the saddle point configuration is asymptotically valid when $T \rightarrow T_{c}$.

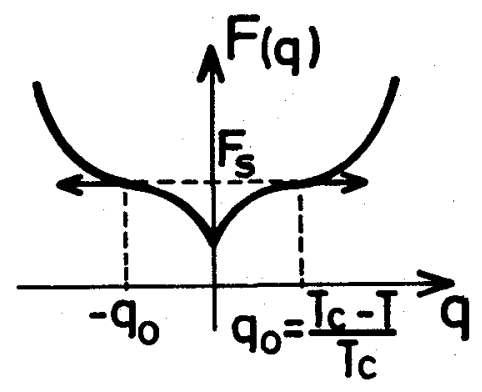

Fig. 14 : The free energy as a function of $q$ below $T_{c}: F\left(q_{o}\right)=2 F(0)$

Within this approach, it is difficult to see clearly a constraint but the same argument than the TAP one should be valid. Also, it has not been possible, for the moment, to find the low temperature properties of the "solvable" model of Sherrington and Kirkpatrick.

c) Conclusion.- We can summarize the main results of the mean field theory for the Ising case :

There is a critical temperature $\mathrm{T}_{c}$ and a new phase below $\mathrm{T}_{c}$. The new phase has characteristic features : the free energy is larger than the analytic continuation of the high temperature free energy ; there are constraints in the fluctuations of the spins which push up the free energy to an extrenum of $F(q)$ which is not a minimum. Near $T_{c}$ at least, there is a characteristic saddle point configuration.

What conclusions about the method of rep1icas ? The spin glass problem is the $n \rightarrow 0$ analytic continuation of the finite $n$ problems. The E A (or $\mathrm{SK}$ ) solution is the continuation of solutions where there is no broken symmetry (Details of the structure of this solution have been studied in details by Almeida and Thouless $/ 41 /$ ). The study of $6 \mathrm{~b}$ ) has a peculiar broken symmetry of the permutation group $\mathrm{s}_{2 \mathrm{~m}}$, which is introduced as a consequence of the definition of $q$ (Eq. (39)). Other broken symmetries could be taken (see Bray and Moore $142 \%$ for example). The difficulty lies in the richness of. the symmetry $s_{n}$. Near $T_{c}$ the most general form of $F$ is:
$F=\frac{k_{B}\left(T_{C}-T\right)}{2} \sum_{\alpha<\beta} q_{\alpha \beta}^{2}-w \sum_{\alpha<\beta<\gamma} q_{\alpha \beta} q_{\beta \gamma} q_{\gamma \alpha}$

and they are many ways of breaking the symmetry.

In the n-vector model of second order phase transitions, the initial symmetry is the rotation group $O(n)$; the broken symmetry is completely specified by the conjugate field, the magnetic field. otherwise, one could have chosen $O(n-2)$ or $0(n-3)$ as broken symmetry group. In this model, the $n \rightarrow 0$ limit is completely specified and it has been very fruitful for the study of polymers. In the case of spin glasses we need something comparable to the magnetic field in order to specify the broken symetry and to allow analytic continuation to $\mathrm{n} \rightarrow 0$. This choice could be (39) and (40).

7. FRUSTATION AND GAUGE THEORIES.- I sha11 say little about these new concepts (as applied to random magnetic systems) and theories because, though certainly going to the heart of the problem they are for the moment in fast evolution, but still at a level which is far from real experiments.

a) Frustration /43/.- The study of competing exchange interactions have been studied since a long time. but the originality of the concept of frustration as introduced by Toulduse /48/ (borrowing the word from Anderson) is its links with other fields of physics (gauge theories).

Consider the Hamiltonian :

$H=-\sum_{(i j)} J_{i j} \vec{s}_{i} \vec{s}_{j}$

with no specifications on the exchange "bonds" $J_{i j}$.

Equation (48) is invariant under the local discrete

transformation (i fixed) :

$\overrightarrow{\mathrm{s}}_{\mathbf{i}} \rightarrow-\overrightarrow{\mathrm{s}}_{\mathbf{i}}$

$J_{i j} \rightarrow-J_{i j}$ for all $j$

Moreover any arbitrary combination of the site transformation (49) leaves equation (48) invariant.

If all $\mathrm{J}_{\mathrm{ij}}$ 's are positive, the transformation (49) introduces an apparent disorder, but this disorder is not very serious (irrelevant) This is the case of the Mattis model /44/: the thermodynamical properties are not changed ; only the susceptibility (response to a uniform magnetic field) is modified because it involves a coupling with the field which is not invariant under the transformation $149 /^{ \pm}$.

This model describes the site randomness (see also ref. /45/ What if of interest here is the bond randomness. 
More serious problems occur when there are competing exchange interactions and they have been recognized since a long time. As a simple minded example let us consider the case of a triangle of Ising spins. The Hamiltonian is :

$H=-J\left(S_{1} S_{2}+S_{2} S_{3}+S_{3} S_{1}\right)$

In the ferromagnetic case $(J>0)$, the ground state has the energy $E_{F}=-3 \mathrm{~J}$ and is doubly degenerate. If $\mathrm{J}<0$, the ground state energy is : $E_{A F}=-|J|$ and it is triply degenerate. This simple example shows clearly two features which have appeared in this review : Competing interactions raise the ground state energy and the ground state degeneracy.

If the spins are n-vectors (Heisenberg for example), the situation is less striking : the spins can have non-parallel directions and the fundamental energy is lower. Thus competing interactions and fructration are fundamentally more serious in the Ising case (discrete group) than for the continuous group of the $n$ vector model ( $n>1$ )

Examples of these remarks are given by the antiferromagnetism in FCC crystals which are built with triangles or by the two dimensional triangular antiferromagnetic Ising model where $\mathrm{T}_{c}=0$.

The conception of frustration has been restricted essentially to nearest neighbour interactions and $( \pm \mathrm{J})$ interactions. Then, another feature is important /43/ : consider for example a square lattice. The interesting quantity is (more than the bonds) the "plaquettes", elementary square cells : the frustration effect is measured by the product of the $J_{i j}$ : $\Phi=\mathrm{J}_{12} \mathrm{~J}_{23} \mathrm{~J}_{34} \mathrm{~J}_{41}$

Toulouse has associated a notion of "curvature" to the plaquettes. If $\Phi=+1$, the plaquette is flat; if $\Phi=-1$ the plaquette is curved. An isolated (-) bond gives rise to two adjacent curved plaquettes (Fig. 15a). An isolated plaquette has a "string" of flipped bonds attached to it. (Fig. 15b). One can construct from the given distribution of bonds the frustration network which acts as sources for defects in the spin system. This model has been used to ca1culate the ground state energy and degeneracy of the square Ising lattice $/ 46 /$.

In the same spirit, Villain /47/ has treated two models of non-random interactions, which exhibit frustration : there is no transition in the one-dimensional Ising spins, whereas there is a phase transition for two dimensional $(x-y)$ spins. The phase transition disappears again for high values of the spin dimensionality $\mathrm{n}$.

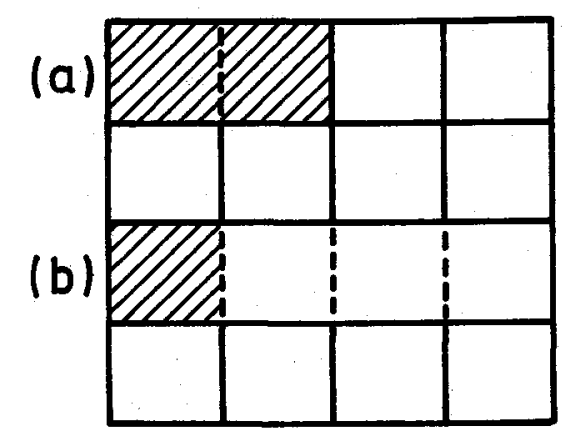

Fig. 15 : Frustration : - positive bond ---- negative bond. The shadded areas correspondant to "frustrated". or "curved" plaquettes.

b) Gauge theories.- The transformation (49) is similar to the invariance which is used in gauge theories. The simplest case of gauge theories is given by the gauge transformation of electrodynamics which modifies the wave function $\phi(\vec{x})$ and the potential vector $\vec{A}(\vec{x})$ as :

$\phi(\vec{x}) \rightarrow e^{i \alpha(\vec{x})} \phi(\vec{x})$

$\vec{A}(\mathrm{x}) \rightarrow \overrightarrow{\mathrm{A}}(\mathrm{x})+\overrightarrow{\mathrm{gr}} \mathrm{ad} \alpha(\overrightarrow{\mathrm{x}})$

In this case the invariance express the conservation

of charge. It is linked with the group $\mathrm{SO}_{2}$ (rotations in two dimensions without reflections), defined by angles $\alpha$, which is a commutative group.

Gauge theories have been introduced in particle physics by Yang and Mills /48/ to describe for example isotropic spin. In general the associated group is non commutative which gives rise to nonlinear effects.

Gauge theories for spin glasses have been developped in two directions :

1) with the discrete Ising group $z_{2}$ which can be handled only on a lattice /49/. The authors do not study the spin glass phase but the frustration network. The main ideas have some similarity with the lattice gauge theories as studied by Wilson /50/ and Balian, Drouffe et Izykson /51/.

2) A continuous version which is valid for continuous spins as described by Dzyaloshinksii and Volovik /52/. The gauge fields (similar to the distortion theory of elasticity) describe "disclinations" in the spin glass system. In this theory, the authors find three longitudinal modes of acoustic type, similar to the Halperin-Saslow modes $/ 53 /^{*}$. Moreover they find spin

* Similar modes (linear in $k$ ) have been found by Edwards and Anderson /54/. 
wave mode behaving as $\mathrm{q}^{3}$ which give a constant density of energy and thus a specific heat linear in $\mathrm{T}$ as observed in experiments and in the simulation of Walker and Waldsedt $/ 13 /$.

Both approaches do not describe the existence of a spin glass transition and its behaviour near $T_{c}$, but they seem to be important for the future description of spin glasses.

\section{RENORMALIZATION.- Renormalization is difficult to} achieve when the mean field theory is not perfect. However, as the free energy shows $\mathrm{T}_{\mathrm{T}}$ ug a cubic term $\mathrm{q}^{3}$ and a linear variation of $q=\frac{\mathrm{c} \mathrm{T}}{\mathrm{T}_{\mathrm{c}}}$ below $\mathrm{T}_{\mathrm{c}}$, the upper critical dimensionality appears to be $d_{c}$ $=6$ (for $\mathrm{d}>6$, classical behaviour is expected $/ 55 /$.

For Ising spins, the spin glass order parameter susceptibility becomes infinite for $d=4$, but the critical temperature does not appear to go to zero /56/. Remark that these dimensionalities 4 and 6 appear in section $6 \mathrm{~b}$ ), where above $T_{c}$, the free energy behave as a $\Phi^{4}$ theory and below $T_{c}$ as a $\Phi^{3}$ theory (Eq. (44) and (45)).

Ising renormalization as been studied in real space by Yound and Stinchcombe /57/. We shall not discuss their approach but let us remark that the lower critical dimensionality appears to be $d_{c}^{m}=2$ for the Ising case. In ordinary phase transitions $d_{c}^{m}=1$, but, as we have seen, the Ising system is the most affected by frustration; this conclusion seems reasonable.

For Heisenberg spins, the lower critical dimensionality could be $d_{c}^{\text {m }}=3$, as suggested by Anderson : this could explain why the dipolar interactions play an important role as shown in section 3, being relevant interactions for this lower critical dimensionality.

A detailed discussion of critical phenomena of random systems is given by Lubensky in ref. $159 /$.

9.CONCLUSION.- A good comparison between experiments and theory is a difficult task today. Let us remark some crucial points :

1) on the experimental point of view, various experiments should be performed with accuracy :

- measurements at low temperatures to set up definitively the linearity (or the non linearity) of the specific heat, and at high temperatures to know the zero-temperature entropy.

- study of the critical point varying the

field but also the frequency $(\mathrm{H} \rightarrow 0, w \rightarrow 0)$
- study of the magnetization jumps and the hysterisis "square" loops : how they appear, why they are asymmetric. Is there a critical field ? 2) On the theoretical point of view, it seems necessary to build a "time dependent" mean field theory, which could be the starting theory to explain the experimental evidences.

On the other hand, the theory as developped itself in various directions, frustration and gauge theories for example. The case of the Ising spin glass (with neighbour interaction), though far from real systems, appears to be "the" model of frustration, interesting by itself as a model of new phase transition. In this direction, simulation experiments as done by Binder and Stauffer $/ 60 /$ are very interesting. I shall not discuss them, as it will be done by Binder at this conference $/ 61 /$

Clearly, exciting problems emerge from the spin glass problem, on the experimental and theoretical points of view : there is a new type of transition and it should be understood, bringing perhaps new concepts. As a simple example let us quote the strange results obtained recently for the magnetization (jumps) and entropy (spikes) of Ising chains and frustrated strips under field which are quite unusual $/ 62 /$.

Mon interêt pour les verres de spin a commencé il y a 20 ans quand avec Friedel nous discutions les distributions de champs moléculaires $P(\xi)$, dessinant diverses possibilitês. Depuis cette date, j'ai eu de multiples discussions intéressantes sur les verres de spin avec de nombreux physiciens et il m'est impossible de les remercier tous. Je ferai exception pour Jacques Friedel qui m'a toujours témoigné sa confiance et éclairé mon travail par son intelligence. 


\section{Références}

/1/ Blandin, A. and Friedel, J., J. Physique Rad. 20 /27/ Löhneysen, H.V., Tholence, J.L. and Tournier, R., (1959) 160.

/2/ Owen, J., Browne, M.E., Knight, W.D. and Kittel, C., Phys. Rev. 102 (1956) 1501 .

/3/ Zimmerman, J, and Hoare, F.E., J. Phys. Chem. Solids 17 (1960) 52.

14/ Blandin, A. Thesis University of Paris (1961) and Lecture Notes, Berkeley (1960).

/5/ Klein, M.W. and Brout, R., Phys. Rev. 132 (1963) 1412 .

16/ Tholence, J.L. and Tournier, R., J. Physique Colloque 5 (1974) C4-229.

17/ Souletie, J., J. Physique, Colloq 39 (1978) C2-3

/8/ Souletie, J., Thesis University of Grenoble (1968)/34/ Sherrington, D: and Kirkpatrick, S., Phys. Rev. Souletie, J. and Tournier, R., J. Low Temp. Phys. 1 (1969) 93.

19/ Marsha11, W., Phys. Rev. 118 (1960) 1519.

/10/ Anderson, P.W., Halperin B.I. and Varma C.M., Philos. Mag. 25 (1972) 1 .

/11/ Villain, J., J. Phys. C.10 (1977) 4793.

/12/ Villain, J., J. Phys. C.11 (1978) 745.

J. Physique, Colloq 39 (1978) C6-922.

/28/ Murani, A.P., Phys. Rev. Lett. 37 (1976) 450.

/29/ Murani, A.P., J. Physique (this issue) .

/30/ Adkins, K. and Rivier, R., J. Physique 35 (1974) C4-237.

131/ Smith, D.A., J. Phys. F.4 (1974) 266. Smith, D.A., J. Phys. F.

132/ Anderson, P.W., Lecture Notes - Summer School of Les Houches (1978). To be published (North Ho11and, 1979).

/33/ Edwards, S.F. and Anderson, P.W., J. Phys. F 5 (1975) 965

/35/ Fisher, H.K., Phys. Rev. Lett. 34 (1975) 1438.

/36/ Blandin, A. (1975) unpublished.

/37/ Kosterlitz, J.M., Thouless, D.J., Jones, R.C., Phys. Rev. Lett. 36 (1976) 1218.

138/ Thouless, D.J., Anderson, P.W. and Palmer, R...., Philos. Mag. 35 (1977) 593.

113/ Walker, L.R. and Waldstedt, R.E., Phys. Rev. Lett./39/ Kirkpatrick, S. and Sherrigton, D., (1978) pre38 (1977) 514

/14/ Schmitt, R.N. and Jacobs, I.S., J. Phys. Chem. Solids $\underline{3}$ (1957) 324 . print.

/15/ Kouvel, J.S., J. Phys. Chem. Solids 21 (1961) 57./41/ Almeida, J.R.L. and Thouless, D.J. (1978) pre-

$116 /$ Lutes, 0.S. and Schmidt, J.L., Phys. Rev. 134 (1964) A. 676.

/17/ Tournier, R., Thesis University of Grenoble (1965).

/18/ Tholence, J.L., Thesis University of Grenoble (1973).

/19/ Tournier, R. and Ishikawa, Y., Phys. Lett. 11 (1964) 280.

/20/ Prējean, J.J., Tholence J.L. and Tournier, R., Private Communication (1977).

/21/ Prêjean, J.J.; J. Physique Colloq 39 (1978) C6907.

/22/ Néel, L., Ann. Geophys. 5 (1949) 99, Néel, L. Physique des Basses Températures (Gordon and Breach, New York, 1961).

Néel, L., Cours de Physique Thểorique - Les Houches (Presses Universitaires de France, Paris, 1961) p. 462 .

/23/ Hobbez, F. and Tholence, J.L., Amorphous Magnetism II (Plenum Press, New York and London, 1977) p. 155 .

/24/ Knitter, R.W., Kouve1, J.S. and Claus, H., J. Mag. Mat. 5 (1977) 356.

/25/ Monod, P. and Préjean, J.J., J. Physique Colloq 39 (1978) C6-910.

/26/ Canella; V. and Mydosh, J.A., Phys. Rev. B. 6 (1972) 4220.

Mydosh, H.A., Amorphous Magnetism II, Levy and Hosegawa, Eds (P1enum Press, 1977) p. 73.

/40/ Blandin, A., Gabay, M. and Garel, T., To be published. print.

/42/ Bray, A.J. and Moore, M.A. (1978) preprint.

143/ Thoulouse, G., Commun. Phys. 2 (1977) 115.

144/ Mattis, D.C., Phys. Lett. 56A (1976) 421.

145/ Bideaux, R., Carton, J.P. and Sarma, G., Phys. Lett. 58A (1976) 467.; Luttinger, J.M., Phys. Rev. L Lett. 37 (1976) 778 .

146/ Vannimenus, J. et Toulouse, G., J. Phys. C10 (1977) L537.

/47/ Villain, J., J. Phys. C 10 (1977) 1717.

/48/ Yang, C.N. and Mills, R.L., Phys. Rev: 96 (1954) 191 .

149/ Fradkin, E., Huberman, B.A. and Shenker, S.H. (1978) preprint.

/50/ Wilson, K.G., Phys. Rev. D10 (1974) 2445.

/51/ B.alian, R., Drouffe, J.M. and Itzykson, C., Phys. Rev. D1.1 (1975) 2098 .

/52/ Dzyaloshinskii, I.E. and Volovik, G.E., J. Physique 39 (1978) 693. Dzyaloshinski, I.E. and Volovik, G.E. (1978) preprint.

153/ Halperin, B.J. and Saslow, W.M., Phys. Rev. B16 (1977) 2154.

154/ Edwards, S.F. and Anderson, P.W., J. Phys. F 6 (1976) 1927.

155/ Harris, A.B., Lubensky, T.G. and Chen, J.H., Phys. Rev. Lett: 36 (1976) 415.

/56/ Fish, R. and Harris, A.B., Phys. Rev. Lett. 38 (1977) 785. 
157/ Young, A.P. and Stinchcombe, R.B., J. Phys. C8 (1975) L535.

Young, A.P. and Stinchcombe, R.B., J. Phys. C 9 (1976) 4419.

Young, A.P., Amorphous Magnetism II (P1enum Press - New York and London, 1977).

158/ Anderson, P.W., Survey of Theories of Spin Glass (1976).

/59/ Lubensky, T.C., Summer School of Les Houches (1978) To be published, (North Holland, 1979).

/60/ Binder, K. and Stauffer, D., Phys. Lett. 57A (1976) 177.

/61/ Binder, K., L T XI, J. Physique To be published (1978).

162/ Derrida, B., Vannimenus, J. and Pomeau, Y., preprint (1978). 